\title{
Enantioselective Synthesis of Bridged- or Fused-Ring Bicyclic Ketones by a Catalytic Asymmetric Michael Addition Pathway
}

\author{
Duan Liu, Sungwoo Hong and E. J. Corey* \\ Department of Chemistry and Chemical Biology \\ Harvard University, 12 Oxford Street, Cambridge, Massachusetts 02138
}

\section{Supplementary Materials}

Materials and Methods. Unless stated otherwise, reactions were performed in flame-dried glassware under a positive pressure of nitrogen using freshly distilled dry solvents. Thin-layer chromatography (TLC) was performed using E. Merck silica gel $60 \mathrm{~F}_{254}$ precoated plates $(0.25$ $\mathrm{mm})$. Flash chromatography was performed using Baker silica gel (40 $\mu \mathrm{m}$ particle size). NMR spectra were recorded on Varian Innova-500, or Mercury-400 instruments and calibrated using residual undeuterated solvent as an internal reference. IR spectra were recorded on Avatar 360 FT-IR spectrometer. Low-resolution and high-resolution mass spectral analyses were performed at the Harvard University Mass Spectrometry Center. Analytical high performance liquid chromatography (HPLC) was performed on Isco 2350 Series or Waters 626 HPLC using the indicated chiral column. Gas chromatography (GC) analyses were performed on HewlettPackard 6850 Series GC System equipped with flame ionization detector using a J \& W Scientific Cyclosil-B column (30 m x $0.25 \mathrm{~mm})$. Commercial grade reagents and solvents were used without further purification except as indicated below. Dichloromethane and $\mathrm{MeCN}$ were distilled from calcium hydride. Toluene, DME and THF were distilled from sodium. 
Oxazaborolidinium Catalyst (S)-5. A 100-mL, two-necked, round-bottomed flask equipped with a stir bar, a glass stopper and a 50-mL pressure-equalizing addition funnel (containing a cotton plug and ca. $10 \mathrm{~g}$ of $4 \mathrm{~A}$ molecular sieves, ${ }^{1}$ and functioning as a Soxhlet extractor) fitted on top with a reflux condenser and a nitrogen inlet adaptor was charged with (S)-(-)- $\alpha, \alpha-$ diphenyl-2-pyrrolidinemethanol (2.59 g, $10.25 \mathrm{mmol}$, from Aldrich or Lancaster), tri-otolylboroxine ${ }^{2}(1.21 \mathrm{~g}, 3.42 \mathrm{mmol})$ and $40 \mathrm{~mL}$ of toluene. The resulting solution was heated to reflux (bath temperature $\sim 145^{\circ} \mathrm{C}$ ). After $3 \mathrm{~h}$, the reaction mixture was cooled to $c a .60{ }^{\circ} \mathrm{C}$ and the addition funnel and condenser were quickly replaced with a short-path distillation head. The mixture was concentrated by distillation (air-cooling) to a volume of ca. $15 \mathrm{~mL}$. This distillation protocol was repeated three times by re-charging with $3 \times 35 \mathrm{~mL}$ of toluene. The solution was then allowed to cool to room temperature and the distillation head was quickly replaced with a vacuum adaptor. Concentration in vacuo (ca. $0.1 \mathrm{mmHg}, 1 \mathrm{~h}$ ) afforded the corresponding oxazaborolidine as clear oil. To an aliquot of the oxazaborolidine precursor $(0.286 \mathrm{mmol}$, theoretical) in toluene $(2.0 \mathrm{~mL})$ at $-25{ }^{\circ} \mathrm{C}$ was added trifluoromethanesulfonimide $(0.10 \mathrm{M}$ solution in toluene, freshly prepared, $2.0 \mathrm{~mL}, 0.20 \mathrm{mmol}$ ) dropwise. After $20 \mathrm{~min}$ at $-20{ }^{\circ} \mathrm{C}$, a colorless homogeneous catalyst solution of (S)-5 was ready for use in the Mukaiyama Michael reactions. The catalyst solution of $(R)-5$ was prepared from $(R)-(+)-\alpha, \alpha$-diphenyl-2pyrrolidinemethanol in a similar manner.

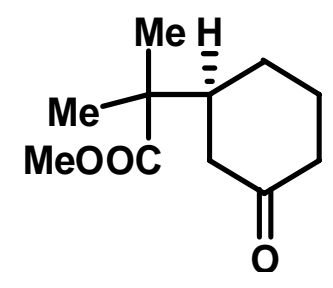

\section{S-6}

Molecular sieves (pellets) were dried in vacuo at ca. $200{ }^{\circ} \mathrm{C}$ with a gas burner for 10 min prior to use.

2 Corey, E. J.; Shibata, T.; Lee, T. W. J. Am. Chem. Soc. 2002, 124, 3808-3809-. 
(S)-(-)-2-Methyl-2-(3-oxocyclohexyl)propionic Acid Methyl Ester (S-6). To a solution of the freshly prepared catalyst $(S)-5(0.05 \mathrm{mmol}, 20 \mathrm{~mol} \%)$ in toluene $(2 \mathrm{~mL})$ were successively added a solution of triphenylphospine oxide $(17.4 \mathrm{mg}, 0.0625 \mathrm{mmol}, 25 \mathrm{~mol} \%)$ in toluene $(0.5 \mathrm{~mL}), 2$ cyclohexen-1-one (2) (24.2 $\mu \mathrm{L}, 0.25 \mathrm{mmol})$, and 1-methoxy-2-methyl-1-(trimethylsilyloxy) propene (4) $(75.8 \mu \mathrm{L}, 0.375 \mathrm{mmol})$ at $-20{ }^{\circ} \mathrm{C}$. The reaction mixture was stirred at $-20{ }^{\circ} \mathrm{C}$ for $16 \mathrm{~h}$ and then quenched by addition of $100 \mu \mathrm{L}$ of $\mathrm{Et}_{3} \mathrm{~N}$. The mixture was warmed to room temperature, and then THF $(5 \mathrm{~mL})$ and aqueous $2 \mathrm{~N} \mathrm{HCl}(1 \mathrm{~mL})$ were carefully added. The resulting mixture was stirred at $23{ }^{\circ} \mathrm{C}$ for $10 \mathrm{~min}$ and extracted with $\mathrm{CH}_{2} \mathrm{Cl}_{2}(3 \times 10 \mathrm{~mL})$. The combined extracts were washed with brine $(10 \mathrm{~mL})$, dried $\left(\mathrm{Na}_{2} \mathrm{SO}_{4}\right)$ and concentrated in vacuo. The residue was purified by flash column chromatography (EtOAc:hexanes, 1:9) to afford (S)-6 (45 mg, 91\%) as a colorless oil: $[\alpha]_{\mathrm{D}}^{25}-20.0$ (c 1.0, $\mathrm{CHCl}_{3}, 90 \%$ ee) lit. $^{3}[\alpha]_{\mathrm{D}}{ }^{25}-14.7$ (c 0.8 , $\mathrm{CHCl}_{3}, 82 \%$ ee); IR (film) 2950, 1713, 1434, 1260, 1194, $1136 \mathrm{~cm}^{-1} ;{ }^{1} \mathrm{H}$ NMR (500 MHz, $\left.\mathrm{CDCl}_{3}\right) \delta 3.63(\mathrm{~s}, 3 \mathrm{H}), 2.37-1.96(\mathrm{~m}, 6 \mathrm{H}), 1.77-1.70(\mathrm{~m}, 1 \mathrm{H}), 1.59-1.50(\mathrm{~m}, 1 \mathrm{H}), 1.38-1.30(\mathrm{~m}$ 1H), $1.13(\mathrm{~s}, 3 \mathrm{H}), 1.11(\mathrm{~s}, 3 \mathrm{H}) ;{ }^{13} \mathrm{C}$ NMR $\left(125 \mathrm{MHz}, \mathrm{CDCl}_{3}\right) \delta 211.1,177.3,51.8,45.8,45.2$, 43.2, 41.1, 26.3, 25.0, 22.0, 21.7. The IR and ${ }^{1} \mathrm{H}$ NMR and ${ }^{13} \mathrm{C}$ NMR spectral data of $(S)-6$ were identical to those reported in the literature for the racemic compound. ${ }^{4}$ Enantioselectivity was determined by HPLC analysis using a Chiralpak IA column (1\% $\mathrm{i}-\mathrm{PrOH}$ in hexanes; 1.0 $\mathrm{mL} / \mathrm{min} ; \lambda 290 \mathrm{~nm}$ ); retention times: $9.8 \mathrm{~min}$ (minor), $11.6 \mathrm{~min}$ (major).

The Michael adduct $(R)-6$ was obtained with the catalyst $(R)-5$ in a similar manner. $[\alpha]_{\mathrm{D}}{ }^{25}+21.8$ (c $1.3, \mathrm{CHCl}_{3}, 90 \%$ ee)

Chordia, M. D.; Harman, W. D. J. Am. Chem. Soc. 2000, 122, 2725-2736.

4 Loh, T.; Wei, L. Tetrahedron 1998, 54, 7615-7624. 


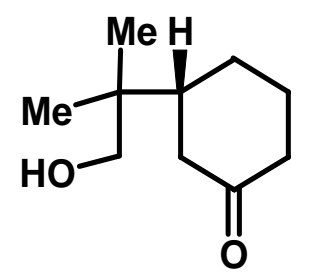

R-7

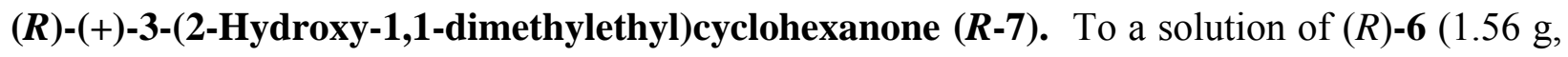
$7.87 \mathrm{mmol})$ in $\mathrm{MeOH}(25 \mathrm{~mL})$ was added trimethyl orthoformate $(1.72 \mathrm{~mL}, 15.7 \mathrm{mmol})$, followed by $p$-TSA $(74.8 \mathrm{mg}, 0.39 \mathrm{mmol})$. The resulting solution was stirred at $23{ }^{\circ} \mathrm{C}$ for $2 \mathrm{~h}$, and then saturated aqueous $\mathrm{NaHCO}_{3}(10 \mathrm{~mL})$ was carefully added. The methanol was removed in vacuo, the resulting residue was extracted with $\mathrm{CH}_{2} \mathrm{Cl}_{2}(3 \times 10 \mathrm{~mL})$. The combined extracts were washed with brine $(10 \mathrm{~mL})$, dried $\left(\mathrm{Na}_{2} \mathrm{SO}_{4}\right)$ and concentrated in vacuo to produce the crude dimethyl ketal $(1.69 \mathrm{~g})$ suitable for use in the next step without further purification.

To a suspension of lithium aluminum hydride $(0.39 \mathrm{~g}, 10.2 \mathrm{mmol})$ in THF $(25 \mathrm{~mL})$ was added dropwise a solution of the above dimethyl ketal $(1.69 \mathrm{~g}, 7.87 \mathrm{mmol})$ in THF $(15 \mathrm{~mL})$ at $0{ }^{\circ} \mathrm{C}$. The reaction mixture was stirred for $2 \mathrm{~h}$ at $0{ }^{\circ} \mathrm{C}$. After stirring for additional $1 \mathrm{~h}$ at 23 ${ }^{\circ} \mathrm{C}, \mathrm{H}_{2} \mathrm{O}(0.4 \mathrm{~mL})$, aqueous $30 \% \mathrm{KOH}(0.4 \mathrm{~mL})$, and $\mathrm{H}_{2} \mathrm{O}(1.2 \mathrm{~mL})$ were consecutively added. The resulting mixture was vigorously stirred for $12 \mathrm{~h}$ and was filtered through a short plug of Celite eluting with THF. The filtrate was concentrated in vacuo, and the residue was dissolved in a mixture of acetone $(20 \mathrm{~mL})$ and aqueous $2 \mathrm{~N} \mathrm{HCl}(2 \mathrm{~mL})$. The resulting mixture was stirred at $23{ }^{\circ} \mathrm{C}$ for $2 \mathrm{~h}$, and the solvent was removed in vacuo. The residue was diluted with brine $(10$ $\mathrm{mL})$ and extracted with $\mathrm{CH}_{2} \mathrm{Cl}_{2}(3 \times 10 \mathrm{~mL})$. The combined extracts were dried $\left(\mathrm{Na}_{2} \mathrm{SO}_{4}\right)$ and concentrated in vacuo. The residue was purified by flash column chromatography (EtOAc:hexanes, 3:7) to afford $(R)-7(1.22 \mathrm{~g}, 91 \%)$ as a colorless oil: $[\alpha]_{\mathrm{D}}^{25}+16.1(c$ 1.0, 
$\mathrm{CHCl}_{3}$ ); IR (film) 3425, 2962, 2875, 1700, 1216, 1048, $751 \mathrm{~cm}^{-1} ;{ }^{1} \mathrm{H}$ NMR $\left(500 \mathrm{MHz}, \mathrm{CDCl}_{3}\right) \delta$ $3.28(\mathrm{~d}, J=1 \mathrm{~Hz}, 2 \mathrm{H}), 2.89(\mathrm{~s}, 1 \mathrm{H}), 2.32-2.24(\mathrm{~m}, 2 \mathrm{H}), 2.19-2.12(\mathrm{~m}, 1 \mathrm{H}), 2.06-1.99(\mathrm{~m}, 2 \mathrm{H})$, $1.83(\mathrm{~d}, J=13 \mathrm{~Hz}, 1 \mathrm{H}), 1.74-1.68(\mathrm{~m}, 1 \mathrm{H}), 1.54-1.44(\mathrm{~m}, 1 \mathrm{H}), 1.34-1.25(\mathrm{~m}, 1 \mathrm{H}) ;{ }^{13} \mathrm{C} \mathrm{NMR}$ $\left(125 \mathrm{MHz}, \mathrm{CDCl}_{3}\right) \delta 213.3,69.6,43.9,42.9,41.1,37.1,25.5,25.4,21.5,21.2$; LRMS (ES+) calcd for $\mathrm{C}_{10} \mathrm{H}_{18} \mathrm{O}_{2}\left(\mathrm{MH}^{+}\right)$171.1, found 171.2.

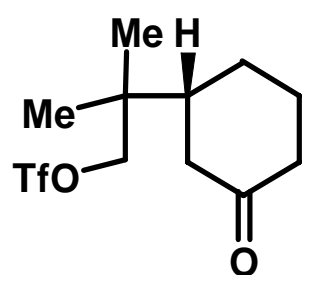

R-8

(R)-(+)-Trifluoromethanesulfonic Acid 2-Methyl-2-(3-oxocyclohexyl)propyl Ester ( $R$ )-8. To a mixture of $(R)-7(325 \mathrm{mg}, 1.91 \mathrm{mmol})$ and 2,6-lutidine $(0.233 \mathrm{~mL}, 2.00 \mathrm{mmol})$ in $\mathrm{CH}_{2} \mathrm{Cl}_{2}(8$ $\mathrm{mL})$ at $0{ }^{\circ} \mathrm{C}$ was added $\mathrm{Tf}_{2} \mathrm{O}(0.336 \mathrm{~mL}, 2.00 \mathrm{mmol})$ dropwise. The reaction mixture was stirred for $1 \mathrm{~h}$ at $0{ }^{\circ} \mathrm{C}$ and then saturated aqueous $\mathrm{NH}_{4} \mathrm{Cl}(5 \mathrm{~mL})$ was carefully added. The mixture was extracted with $\mathrm{CH}_{2} \mathrm{Cl}_{2}(3 \times 5 \mathrm{~mL})$. The combined extracts were washed with saturated aqueous $\mathrm{NaHCO}_{3}(10 \mathrm{~mL})$, brine $(5 \mathrm{~mL})$, dried $\left(\mathrm{Na}_{2} \mathrm{SO}_{4}\right)$ and concentrated in vacuo. The residue was purified by flash column chromatography (EtOAc:hexanes, 1:5) to afford (R)-8 (420 mg, 73\%) as a pale pink oil: $[\alpha]_{\mathrm{D}}^{25}+11.2\left(\right.$ ( $\left.1.0, \mathrm{CHCl}_{3}\right)$; IR (film) $2970,2875,1713,1410,1246,1202$, 1144, 930, $851 \mathrm{~cm}^{-1} ;{ }^{1} \mathrm{H}$ NMR (500 MHz, $\left.\mathrm{CDCl}_{3}\right) \delta 3.28(\mathrm{dd}, J=9.5,29.0 \mathrm{~Hz}, 2 \mathrm{H}), 2.42-2.38$ (m, 2H), 2.28-2.21 (m, 1H), 2.17-2.07 (m, 2H), 1.92-1.88 (m, 1H), 1.81-1.74 (m, 1H), 1.64-1.54 (m, 1H), 1.44-1.36 (m, 1H); ${ }^{13} \mathrm{C}$ NMR $\left(125 \mathrm{MHz}, \mathrm{CDCl}_{3}\right) \delta 210.6,118.6(\mathrm{q}, J=318 \mathrm{~Hz}), 82.8$ 43.8, 42.6, 41.0, 37.0, 25.6, 25.0, 21.5, 20.8; LRMS (ES+) calcd for $\mathrm{C}_{11} \mathrm{H}_{19} \mathrm{~F}_{3} \mathrm{O}_{5} \mathrm{~S}\left(\mathrm{M}+\mathrm{H}_{2} \mathrm{O}^{+}\right)$ 302.1, found 302.1. 


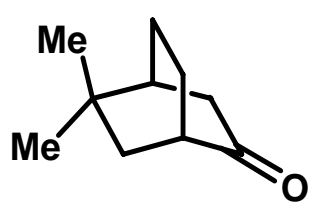

R-9

$(\boldsymbol{R})-(-)-5,5-D i m e t h y l b i c y c l o[2.2 .2]$ octan-2-one $(\boldsymbol{R}-9)$. To a solution of $(R)-8(121 \mathrm{mg}, 0.4$ $\mathrm{mmol})$ in THF $(4.0 \mathrm{~mL})$ at $-78^{\circ} \mathrm{C}$ was added dropwise a solution of KHMDS $(0.5 \mathrm{M}$ in toluene, $0.8 \mathrm{~mL}$ ). The reaction mixture was stirred at $-78^{\circ} \mathrm{C}$ for $30 \mathrm{~min}$ and then quenched by addition of saturated aqueous $\mathrm{NH}_{4} \mathrm{Cl}(0.1 \mathrm{~mL})$. After warmed to room temperature, the $\mathrm{THF}$ was removed in vacuo. The residue was diluted with $\mathrm{H}_{2} \mathrm{O}(5 \mathrm{~mL})$ and extracted with $\mathrm{CH}_{2} \mathrm{Cl}_{2}(3 \times 10 \mathrm{~mL})$. The combined extracts were washed with brine $(5 \mathrm{~mL})$, dried $\left(\mathrm{Na}_{2} \mathrm{SO}_{4}\right)$ and concentrated in vacuo. The residue was purified by flash column chromatography (ether:pentane, 1:5) to afford (R)-9 (49 mg, 80\%) as a colorless oil: $[\alpha]_{\mathrm{D}}^{25}-37.4$ (c 1.0, $\mathrm{CHCl}_{3}$ ); IR (film) 2950, 2869, 1727, 1088 $\mathrm{cm}^{-1} ;{ }^{1} \mathrm{H}$ NMR $\left(500 \mathrm{MHz}, \mathrm{CDCl}_{3}\right) \delta$ 2.57-2.52 (m, 1H), 2.24-2.22 (m, 1H), 2.10-2.00 (m, 2H), 1.77-1.69 (m, 3H), 1.60-1.46 (m, 3H), $1.12(\mathrm{~s}, 3 \mathrm{H}), 0.98(\mathrm{~s}, 3 \mathrm{H}) ;{ }^{13} \mathrm{C} \mathrm{NMR}\left(125 \mathrm{MHz}, \mathrm{CDCl}_{3}\right) \delta$ $217.9,44.2,42.1,39.9,39.0,30.6,30.2,29.9,21.9,21.8$; LRMS (ES+) calcd for $\mathrm{C}_{10} \mathrm{H}_{17} \mathrm{O}\left(\mathrm{MH}^{+}\right)$ 153.1, found 153.1 .

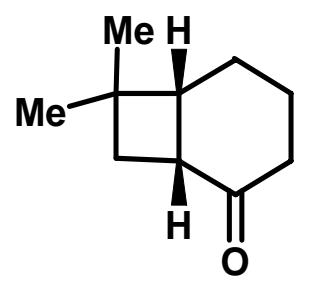

R-3

$(\boldsymbol{R})-(+)-7,7-D i m e t h y l b i c y c l o[4.2 .0]$ octan-2-one $(\boldsymbol{R}-3)$. To a solution of $(R)-8(151 \mathrm{mg}, 0.5$ $\mathrm{mmol})$ in isopropanol $(1.0 \mathrm{~mL})$ at $-50{ }^{\circ} \mathrm{C}$ was added $\mathrm{TMSCl}(64 \mu \mathrm{L}, 0.5 \mathrm{mmol})$, followed by 
pyrrolidine $(125 \mu \mathrm{L}, 1.5 \mathrm{mmol})$. The resulting homogeneous mixture was kept at $-50{ }^{\circ} \mathrm{C}$ for $48 \mathrm{~h}$ and then quenched by addition of saturated aqueous $\mathrm{NH}_{4} \mathrm{Cl}(0.1 \mathrm{~mL})$ at the same temperature. After warmed to room temperature, the reaction mixture was diluted with $\mathrm{H}_{2} \mathrm{O}(5 \mathrm{~mL})$ and extracted with $\mathrm{CH}_{2} \mathrm{Cl}_{2}(3 \times 5 \mathrm{~mL})$. The combined extracts were washed with brine $(5 \mathrm{~mL})$, dried $\left(\mathrm{Na}_{2} \mathrm{SO}_{4}\right)$ and concentrated in vacuo. The residue was purified by flash column chromatography (ether:pentane, $1: 5)$ to afford $(R)-3(55 \mathrm{mg}, 72 \%)$ as a colorless oil: $[\alpha]_{\mathrm{D}}{ }^{25}-105.2\left(c 0.8, \mathrm{CHCl}_{3}\right)$; IR (film) 2939, 2865, 1702, $1457 \mathrm{~cm}^{-1} ;{ }^{1} \mathrm{H}$ NMR $\left(500 \mathrm{MHz}, \mathrm{CDCl}_{3}\right) \delta 2.96$ (q, $\left.J=8.8 \mathrm{~Hz}, 1 \mathrm{H}\right)$, $2.43(\mathrm{dq}, J=1,8.8 \mathrm{~Hz}, 1 \mathrm{H}), 2.36-2.33(\mathrm{~m}, 2 \mathrm{H}), 2.03-1.96(\mathrm{~m}, 3 \mathrm{H}), 1.79-1.71(\mathrm{~m}, 2 \mathrm{H}), 1.65-1.58$ (m, 1H), $1.16(\mathrm{~s}, 3 \mathrm{H}), 0.98(\mathrm{~s}, 3 \mathrm{H}) ;{ }^{13} \mathrm{C} \mathrm{NMR}\left(125 \mathrm{MHz}, \mathrm{CDCl}_{3}\right) \delta$ 215.6, 45.0, 39.47, 39.46, 37.3, 36.1, 29.8, 23.8, 22.9, 22.6; LRMS (ES+) calcd for $\mathrm{C}_{10} \mathrm{H}_{17} \mathrm{O}\left(\mathrm{MH}^{+}\right)$153.1, found 153.1. Selectivity $(R-3 / R-9,10: 1)$ was determined by ${ }^{1} \mathrm{H}$ NMR $\left(500 \mathrm{MHz}, \mathrm{CDCl}_{3}\right)$ analysis of the crude mixture: $\delta 1.16(\mathrm{~s}, R-3), \delta 1.13(\mathrm{~s}, R-9)$.

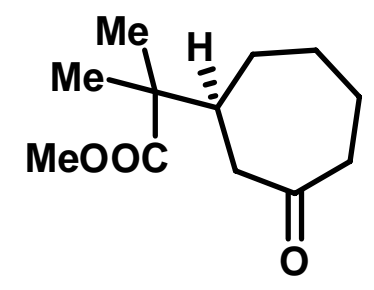

11

(S)-(-)-2-Methyl-2-(3-oxocycloheptyl)propionic Acid Methyl Ester (11). To a solution of the freshly prepared catalyst $(S)-5(0.05 \mathrm{mmol}, 20 \mathrm{~mol} \%)$ in toluene $(2 \mathrm{~mL})$ were successively added a solution of triphenylphospine oxide $(17.4 \mathrm{mg}, 0.0625 \mathrm{mmol}, 25 \mathrm{~mol} \%)$ in toluene $(0.5 \mathrm{~mL})$, 2,6-diisopropylphenol (46.3 $\mu \mathrm{L}, 0.25 \mathrm{mmol})$, 2-cyclohepten-1-one (10) $(34.8 \mu \mathrm{L}, 0.25 \mathrm{mmol})$, and 1-methoxy-2-methyl-1-(trimethylsilyloxy)propene (4) $(152 \mu \mathrm{L}, 0.75 \mathrm{mmol})$ at $-20{ }^{\circ} \mathrm{C}$. The reaction mixture was stirred at the same temperature for $6 \mathrm{~h}$ and then quenched by addition of 
$100 \mu \mathrm{L}$ of $\mathrm{Et}_{3} \mathrm{~N}$. The mixture was warmed to room temperature, and then THF $(5 \mathrm{~mL})$ and aqueous $2 \mathrm{~N} \mathrm{HCl}(1 \mathrm{~mL})$ were carefully added. The resulting mixture was stirred at $23{ }^{\circ} \mathrm{C}$ for 10 min and extracted with $\mathrm{CH}_{2} \mathrm{Cl}_{2}(3 \times 10 \mathrm{~mL})$. The combined extracts were washed with brine $(10$ $\mathrm{mL})$, dried $\left(\mathrm{Na}_{2} \mathrm{SO}_{4}\right)$ and concentrated in vacuo. The residue was purified by flash column chromatography (EtOAc:hexanes, 1:9) to afford the desired ester 11 (53 mg, 99\%) as a colorless oil: $[\alpha]_{\mathrm{D}}{ }^{25}-68.7$ (c 1.0, $\mathrm{CHCl}_{3}, 99 \%$ ee); IR (film) 2933, 1729, 1702, 1447, 1246, $1144 \mathrm{~cm}^{-1} ;{ }^{1} \mathrm{H}$ NMR (400 MHz, $\left.\mathrm{CDCl}_{3}\right) \delta 3.67(\mathrm{~s}, 3 \mathrm{H}), 2.58-2.32(\mathrm{~m}, 4 \mathrm{H}), 2.10-1.85(\mathrm{~m}, 3 \mathrm{H}), 1.76-1.70(\mathrm{~m}$, 1H), $1.60-1.49(\mathrm{~m}, 1 \mathrm{H}), 1.42-1.32(\mathrm{~m}, 1 \mathrm{H}), 1.23-1.13(\mathrm{~m}, 1 \mathrm{H}), 1.12(\mathrm{~s}, 3 \mathrm{H}), 1.11(\mathrm{~s}, 3 \mathrm{H}) ;{ }^{13} \mathrm{C}$ $\operatorname{NMR}\left(100 \mathrm{MHz}, \mathrm{CDCl}_{3}\right) \delta$ 213.9, 177.9, 51.9, 46.5, 45.6, 43.4, 42.7, 32,2, 29.3, 24.8, 22.4, 21.1; HRMS (ES+) calcd for $\mathrm{C}_{12} \mathrm{H}_{21} \mathrm{O}_{3}\left(\mathrm{MH}^{+}\right)$213.1490, found 213.1485. Enantioselectivity was determined by HPLC analysis using a Chiralcel OD-H column $(1 \%$ i-PrOH in hexanes; 1.0 $\mathrm{mL} / \mathrm{min} ; \lambda 290 \mathrm{~nm}$ ); retention times: $7.1 \mathrm{~min}$ (minor), $8.5 \mathrm{~min}$ (major).

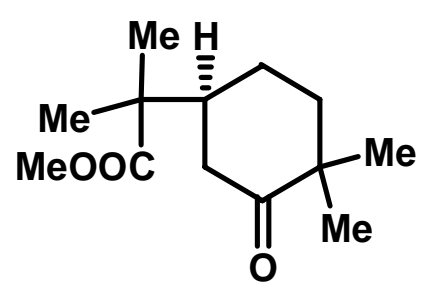

12

(S)-(-)-2-(4,4-Dimethyl-3-oxocyclohexyl)-2-methylpropionic Acid Methyl Ester (12). This compound was obtained from 1-methoxy-2-methyl-1-(trimethylsilyloxy)propene (4) and 6,6dimethyl 2-cyclohexen-1-one in a similar manner to $\mathbf{1 1}$ as a colorless oil $\left(80 \%\right.$ yield): $[\alpha]_{\mathrm{D}}{ }^{25}$ -91.9 (c 1.0, $\mathrm{CHCl}_{3}, 92 \%$ ee); IR (film) 2973, 2950, 1729, 1708, 1463, 1266, 1191, 1146, 1123 $\mathrm{cm}^{-1} ;{ }^{1} \mathrm{H}$ NMR $\left(400 \mathrm{MHz}, \mathrm{CDCl}_{3}\right) \delta 3.66(\mathrm{~s}, 3 \mathrm{H}), 2.38(\mathrm{t}, J=13.6 \mathrm{~Hz}, 1 \mathrm{H}), 2.19-2.14(\mathrm{~m}, 1 \mathrm{H})$, 2.03-1.95 (m, 1H), 1.78-1.73 (m, 1H), 1.65-1.47 (m, 3H), $1.16(\mathrm{~s}, 3 \mathrm{H}), 1.14(\mathrm{~s}, 3 \mathrm{H}), 1.12(\mathrm{~s}, 3 \mathrm{H})$, 
$1.03(\mathrm{~s}, 3 \mathrm{H}) ;{ }^{13} \mathrm{C}$ NMR $\left(100 \mathrm{MHz}, \mathrm{CDCl}_{3}\right) \delta 215.6,177.6,52.1,46.7,45.5,44.7,39.8,39.7$, 25.21, 25.20, 22.9, 22.5, 22.1; HRMS (ES+) calcd for $\mathrm{C}_{13} \mathrm{H}_{23} \mathrm{O}_{3}\left(\mathrm{MH}^{+}\right)$227.1647, found 227.1637. Enantioselectivity was determined by HPLC analysis using a Chiralpak IA column (0.5\% $\mathrm{i}-\mathrm{PrOH}$ in hexanes; $1.0 \mathrm{~mL} / \mathrm{min} ; \lambda 290 \mathrm{~nm}$ ); retention times: $6.3 \mathrm{~min}$ (minor), $6.9 \mathrm{~min}$ (major).

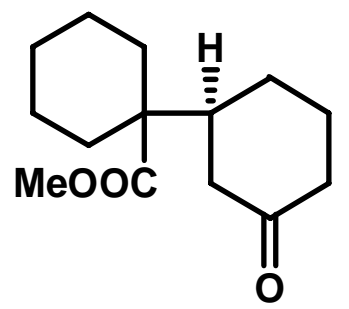

13

(S)-(-)-3'-Oxobicyclohexyl-1-carboxylic Acid Methyl Ester (13). This compound was obtained from [cyclohexylidene(methoxy)methoxy]trimethylsilane and 2-cyclohexen-1-one (2) in a similar manner to $\mathbf{1 1}$ as a colorless oil (86\% yield): $[\alpha]_{\mathrm{D}}{ }^{25}-7.0\left(\right.$ c $1.7, \mathrm{CHCl}_{3}, 90 \%$ ee); IR (film) 2958, 2925, 2860, 1721, 1455, 1200, $1133 \mathrm{~cm}^{-1}$; ${ }^{1} \mathrm{H}$ NMR $\left(500 \mathrm{MHz}, \mathrm{CDCl}_{3}\right) \delta 3.69(\mathrm{~s}$, 3H), 2.43-2.31 (m, 2H), 2.23-2.03 (m, 5H), 1.93-1.88 (m, 1H), 1.81-1.73 (m, 1H), 1.65-1.45 (m, 4H), 1.34-1.07 (m, 6H); ${ }^{13} \mathrm{C}$ NMR (125 MHz, $\left.\mathrm{CDCl}_{3}\right) \delta 211.5,175.5,51.4,50.4,47.1,43.4,41.3$, $31.8,31.5,26.3,25.7,25.2,23.54,23.52$; HRMS (ES+) calcd for $\mathrm{C}_{14} \mathrm{H}_{23} \mathrm{O}_{3}\left(\mathrm{MH}^{+}\right) 239.1647$ found 239.1641. Enantioselectivity was determined by HPLC analysis using a Chiralcel OD-H column (1\% $i$-PrOH in hexanes; $1.0 \mathrm{~mL} / \mathrm{min} ; \lambda 290 \mathrm{~nm})$; retention times: $9.8 \mathrm{~min}$ (major), 12.1 $\min ($ minor). 


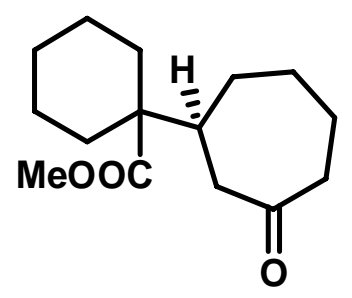

14

S)-(-)-1-(3-Oxocycloheptyl)cyclohexanecarboxylic Acid Methyl Ester (14). This compound was obtained from [cyclohexylidene(methoxy)methoxy]trimethylsilane and 2-cyclohepten-1-one (10) in a similar manner as a colorless oil (89\% yield): $[\alpha]_{\mathrm{D}}^{25}-43.0$ (c 1.0, $\mathrm{CHCl}_{3}, 88 \%$ ee); IR (film) 2929, 2858, 1725, 1702, 1451, 1200, $1131 \mathrm{~cm}^{-1} ;{ }^{1} \mathrm{H} \mathrm{NMR}\left(400 \mathrm{MHz}, \mathrm{CDCl}_{3}\right) \delta 3.68(\mathrm{~s}$, $3 \mathrm{H}), 2.53-2.35(\mathrm{~m}, 4 \mathrm{H}), 2.08-1.86(\mathrm{~m}, 5 \mathrm{H}), 1.78-1.71(\mathrm{~m}, 1 \mathrm{H}), 1.64-1.49(\mathrm{~m}, 4 \mathrm{H}), 1.34-1.06(\mathrm{~m}$, $7 \mathrm{H}) ;{ }^{13} \mathrm{C} \mathrm{NMR}\left(100 \mathrm{MHz}, \mathrm{CDCl}_{3}\right) \delta 214.1,175.9,51.4,51.0,45.2,44.2,43.2,31.5,31.3,30.9$, 29.1, 25.7, 24.7, 23.44, 23.40; HRMS (ES+) calcd for $\mathrm{C}_{15} \mathrm{H}_{25} \mathrm{O}_{3}\left(\mathrm{MH}^{+}\right) 253.1803$ found 253.1807. Enantioselectivity was determined by HPLC analysis using a Chiralcel OJ column $(1 \% \mathrm{i}-\mathrm{PrOH}$ in hexanes; $1.0 \mathrm{~mL} / \mathrm{min} ; \lambda 290 \mathrm{~nm})$; retention times: 9.0 min (major), $11.2 \mathrm{~min}$ (minor).

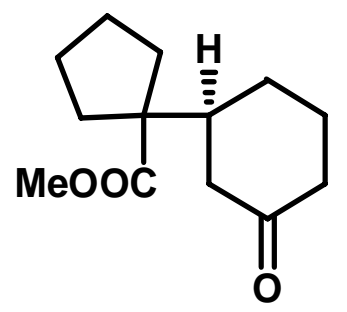

15

(S)-(-)-1-(3-Oxocyclohexyl)cyclopentanecarboxylic Acid Methyl Ester (15). To a solution of the freshly prepared catalyst $(S)-5(0.05 \mathrm{mmol}, 20 \mathrm{~mol} \%)$ in toluene $(1 \mathrm{~mL})$ was added a solution of triphenylphospine oxide $(17.4 \mathrm{mg}, 0.0625 \mathrm{mmol}, 25 \mathrm{~mol} \%)$ in toluene $(0.5 \mathrm{~mL})$, followed by 
[cyclopentylidene(methoxy)methoxy] trimethylsilane $(134 \mu \mathrm{L}, 0.625 \mathrm{mmol})$ at $-20{ }^{\circ} \mathrm{C}$. A solution in toluene (1 mL) of 2-cyclohexen-1-one (2) $(24.2 \mu \mathrm{L}, 0.25 \mathrm{mmol})$ and 2,6-diisopropylphenol $(46.3 \mu \mathrm{L}, 0.25 \mathrm{mmol})$ was then slowly added via a syringe drive over $4 \mathrm{~h}$ at $-20{ }^{\circ} \mathrm{C}$. After addition, the reaction mixture was stirred at the same temperature for $12 \mathrm{~h}$ and then quenched by addition of $100 \mu \mathrm{L}$ of $\mathrm{Et}_{3} \mathrm{~N}$. The mixture was warmed to room temperature, and then THF (5 $\mathrm{mL})$ and aqueous $2 \mathrm{~N} \mathrm{HCl}(1 \mathrm{~mL})$ were carefully added. The resulting mixture was stirred at 23 ${ }^{\circ} \mathrm{C}$ for $10 \mathrm{~min}$ and extracted with $\mathrm{CH}_{2} \mathrm{Cl}_{2}(3 \times 10 \mathrm{~mL})$. The combined extracts were washed with brine $(10 \mathrm{~mL})$, dried $\left(\mathrm{Na}_{2} \mathrm{SO}_{4}\right)$ and concentrated in vacuo. The residue was purified by flash column chromatography (EtOAc:hexanes, 1:9) to afford 15 as a colorless oil (44 $\mathrm{mg}, 79 \%$ ): $[\alpha]_{\mathrm{D}}^{25}-2.8$ (c 1.0, $\mathrm{CHCl}_{3}, 82 \%$ ee); IR (film) 2952, 2871, 1713, 1451, 1233, 1194, $1162 \mathrm{~cm}^{-1} ;{ }^{1} \mathrm{H}$ NMR (400 MHz, $\left.\mathrm{CDCl}_{3}\right) \delta 3.68(\mathrm{~s}, 3 \mathrm{H}), 2.42-2.32(\mathrm{~m}, 2 \mathrm{H}), 2.26-1.94(\mathrm{~m}, 6 \mathrm{H}), 1.90-1.83(\mathrm{~m}$, 1H), 1.63-1.32 (m, 8H); ${ }^{13} \mathrm{C}$ NMR $\left(100 \mathrm{MHz}, \mathrm{CDCl}_{3}\right) \delta 211.2,176.8,58.1,51.8,46.3,44.7,41.2$, 33.9, 33.7, 27.8, 25.2, 24.83, 24.76; HRMS (ES+) calcd for $\mathrm{C}_{13} \mathrm{H}_{21} \mathrm{O}_{3}\left(\mathrm{MH}^{+}\right) 225.1490$ found 225.1492. Enantioselectivity was determined by HPLC analysis using a Chiralcel OD-H column (0.5\% $\mathrm{i}$-PrOH in hexanes; $1.0 \mathrm{~mL} / \mathrm{min} ; \lambda 290 \mathrm{~nm}$ ); retention times: $13.3 \mathrm{~min}$ (major), $15.3 \mathrm{~min}$ (minor).

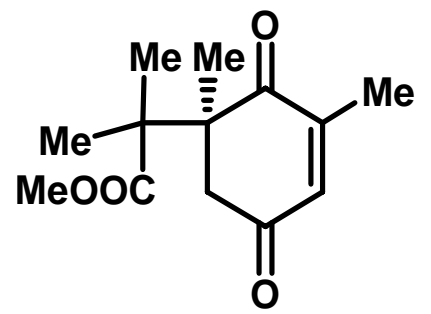


(S)-(-)-2-(1,3-Dimethyl-2,5-dioxocyclohex-3-enyl)-2-methyl-propionic Acid Methyl Ester

(17). This compound was obtained from 1-methoxy-2-methyl-1-(trimethylsilyloxy)propene (4) and 2,6-dimethylbenzoquinone (16) in a similar manner as a colorless oil $(87 \%$ yield $):[\alpha]_{\mathrm{D}}^{25}$ -15.2 (c 1.0, $\mathrm{CHCl}_{3}, 90 \%$ ee); IR (film) 2985, 2954, 1723, 1675, 1434, 1272, 1191, $1144 \mathrm{~cm}^{-1}$;

${ }^{1} \mathrm{H}$ NMR $\left(300 \mathrm{MHz}, \mathrm{CDCl}_{3}\right) \delta 6.54(\mathrm{dd}, J=0.9,1.2 \mathrm{~Hz}, 1 \mathrm{H}), 3.63(\mathrm{~s}, 3 \mathrm{H}), 3.35(\mathrm{~d}, J=16.2 \mathrm{~Hz}$, 1H), 2.59 (dd, $J=0.9,16.2 \mathrm{~Hz}, 1 \mathrm{H}), 1.99$ (d, $J=1.2 \mathrm{~Hz}, 3 \mathrm{H}), 1.40(\mathrm{~s}, 3 \mathrm{H}), 1.24(\mathrm{~s}, 3 \mathrm{H}), 1.18$ (s, $3 \mathrm{H}) ;{ }^{13} \mathrm{C} \mathrm{NMR}\left(100 \mathrm{MHz}, \mathrm{CDCl}_{3}\right) \delta 202.1,197.5,177.2,150.2,136.3,51.9,51.3,48.3,48.1$, 22.5, 22.1, 21.9, 16.9; LRMS (ES+) calcd for $\mathrm{C}_{13} \mathrm{H}_{19} \mathrm{O}_{4}\left(\mathrm{MH}^{+}\right) 239.1$ found 239.1. Enantioselectivity was determined by HPLC analysis using a Chiralcel OJ column $(1 \% \mathrm{i}-\mathrm{PrOH}$ in hexanes; $1.0 \mathrm{~mL} / \mathrm{min} ; \lambda 254 \mathrm{~nm}$ ); retention times: 20.7 min (major), $25.2 \mathrm{~min}$ (minor).

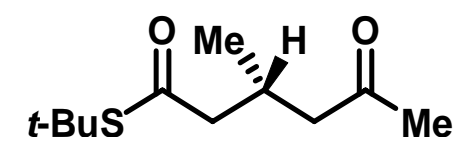

21

(S)-(-)-tert-Butyl (R)-3-Methyl-5-oxohexanethioate (21). To a solution of the freshly prepared catalyst $(S)-5(0.05 \mathrm{mmol}, 20 \mathrm{~mol} \%)$ of toluene $(2 \mathrm{~mL})$ were successively added a solution of triphenylphospine oxide (17.4 $\mathrm{mg}, 0.0625 \mathrm{mmol}, 25 \mathrm{~mol} \%)$ in toluene $(0.5 \mathrm{~mL}), 2,6-$ diisopropylphenol (139 $\mu \mathrm{L}, 0.75 \mathrm{mmol})$, pent-3-en-2-one (19) $(27.0 \mu \mathrm{L}, 0.25 \mathrm{mmol})$, and (1-tertbutylsulfanylvinyloxy)trimethylsilane (18) $(173 \mu \mathrm{L}, 0.75 \mathrm{mmol})$ at $-78{ }^{\circ} \mathrm{C}$. The reaction mixture was stirred at $-78{ }^{\circ} \mathrm{C}$ for $24 \mathrm{~h}$ and then quenched by addition of $100 \mu \mathrm{L}$ of $\mathrm{Et}_{3} \mathrm{~N}$. The mixture was warmed to room temperature, and then THF $(5 \mathrm{~mL})$ and aqueous $6 \mathrm{~N} \mathrm{HCl}(1 \mathrm{~mL})$ were carefully added. The resulting mixture was stirred at $23{ }^{\circ} \mathrm{C}$ for $10 \mathrm{~min}$ and extracted with $\mathrm{CH}_{2} \mathrm{Cl}_{2}(3 \times 10 \mathrm{~mL})$. The combined extracts were washed with brine $(10 \mathrm{~mL})$, dried $\left(\mathrm{Na}_{2} \mathrm{SO}_{4}\right)$ 
and concentrated in vacuo. The residue was purified by flash column chromatography (EtOAc:hexanes, 1:9) to afford the desired ester 21 (53 mg, 99\%) as a colorless oil: $[\alpha]_{\mathrm{D}}{ }^{25}-5.7$ (c 1.0, $\mathrm{CHCl}_{3}, 90 \%$ ee); ${ }^{1} \mathrm{H}$ NMR (500 MHz, $\left.\mathrm{CDCl}_{3}\right) \delta 2.54-2.41(\mathrm{~m}, 3 \mathrm{H}), 2.36-2.24(\mathrm{~m}, 2 \mathrm{H})$, $2.12(\mathrm{~s}, 3 \mathrm{H}), 1.44(\mathrm{~s}, 9 \mathrm{H}), 0.96(\mathrm{~d}, J=6.5 \mathrm{~Hz}, 3 \mathrm{H}) ;{ }^{13} \mathrm{C} \mathrm{NMR}\left(125 \mathrm{MHz}, \mathrm{CDCl}_{3}\right) \delta$ 207.7, 199.4, 50.6, 49.7, 48.0, 30.3, 29.7, 27.1, 19.7 (The ${ }^{1} \mathrm{H}$ NMR and ${ }^{13} \mathrm{C}$ NMR spectral data were identical to those reported in the literature). ${ }^{5}$ Enantioselectivity was determined by HPLC analysis using a Chiralcel AD column ( $0.5 \%$ i-PrOH in hexanes; $0.5 \mathrm{~mL} / \mathrm{min} ; \lambda 254 \mathrm{~nm})$; retention times: $9.8 \mathrm{~min}$ (major), $10.6 \min$ (minor).

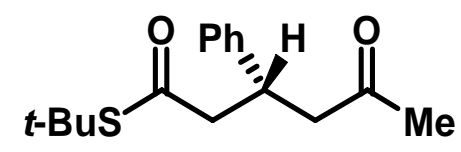

22

(S)-(-)-tert-Butyl (R)-3-Phenyl-5-oxohexanethioate (22). This compound was obtained from 4-phenyl-3-buten-2-one (20) (36.5 $\mathrm{mg}, \quad 0.25 \mathrm{mmol}$ ) and (1-tert-butylsulfanylvinyloxy) trimethylsilane (18) $(173 \mu \mathrm{L}, 0.75 \mathrm{mmol})$ in a manner similar to 21 as a white solid (99\% yield): $[\alpha]_{\mathrm{D}}{ }^{25}-29.4\left(c \mathrm{1} .0, \mathrm{CHCl}_{3}, 84 \%\right.$ ee); ${ }^{1} \mathrm{H} \mathrm{NMR}\left(400 \mathrm{MHz}, \mathrm{CDCl}_{3}\right) \delta$ 7.30-7.26 (m, 2H), 7.21-7.17 (m, 3H), 3.70 (quintet, $J=7.2 \mathrm{~Hz}, 1 \mathrm{H}), 2.87-2.68(\mathrm{~m}, 4 \mathrm{H}), 2.04(\mathrm{~s}, 3 \mathrm{H}), 1.38(\mathrm{~s}, 9 \mathrm{H}) ;{ }^{13} \mathrm{C} \mathrm{NMR}$ $\left(100 \mathrm{MHz}, \mathrm{CDCl}_{3}\right) \delta 206.7,198.5,142.6,128.5,127.4,126.8,50.4,49.0,48.1,38.0,30.3,29.6$ (The ${ }^{1} \mathrm{H}$ NMR and ${ }^{13} \mathrm{C}$ NMR spectral data were identical to those reported in the literature). ${ }^{5}$ Enantioselectivity was determined by HPLC analysis using a Chiralcel AD column $(0.5 \%$ iPrOH in hexanes; $0.5 \mathrm{~mL} / \mathrm{min} ; \lambda 254 \mathrm{~nm}$ ); retention times: $20.6 \mathrm{~min}$ (major), $24.0 \mathrm{~min}$ (minor).

\footnotetext{
5 Wang, X.; Adachi, S.; Iwai, H.; Takatsuki, H.; Fujita, K.; Kubo, M.; Oku, A.; Harada, T. J. Org. Chem. 2003, 68, 10046-10057.
} 